\title{
Instantaneous determination of a variable extinction coefficient in photoelectric photometry
}

\author{
E. Poretti ${ }^{1}$, F. Zerbi ${ }^{2}$ \\ ${ }^{1}$ Osservatorio Astronomico di Brera, Merate, Italy, ${ }^{2}$ Dipartimento di Fisica \\ Nucleare e Teorica, Università di Pavia, Italy
}

\begin{abstract}
Observational evidence was found that even in good photometric sites the extinction coefficient can display large variations during a night. The authors propose a method to determine instantaneous values of the extinction coefficient: it is based on the knowledge of the extra-atmosphere instrumental magnitude $m_{o}$ for a reference star. An analytical procedure is proposed to determine a reliable value for $m_{o}$ and results are very satisfactory.
\end{abstract}

\section{Introduction}

At the effective wavelength $\lambda$, the ground observed instrumental magnitude $m$ is related to the extra-atmosphere instrumental magnitude $m_{o}$ by Bouguer's law

$$
m=m_{\circ}+k_{\lambda}^{\prime} \sec z
$$

where $k_{\lambda}^{\prime}$ is the extinction coefficient (measured in mag/airmass) and $\sec z$ is a good approximation for the airmass value. When expressing the magnitude difference between two stars we have

$$
\Delta m=\Delta m_{o}+k_{\lambda}^{\prime} \Delta \sec z+k_{\lambda}^{\prime \prime} \Delta(B-V) \overline{\sec z}
$$

where $k_{\lambda}^{\prime}$ is the first-order coefficient and $k_{\lambda}^{\prime \prime}$ the second-order coefficient; in this paper we shall deal with $U B V$ photometry and hence we use $B-V$ as colour index. Very accurate differential measurements can be performed in not high-quality photometric conditions by choosing the comparison stars among the stars not only located close to the program star but also having a very similar colour index; the only request is a regular variability of the sky transparency over an observing cycle. However, when searching for small amplitude variations, the contribution of the product $k_{\lambda}^{\prime} \Delta \sec z$ can rarely be considered to be negligible and a precise determination of $k_{\lambda}^{\prime}$ is necessary.

In the data reduction, the extinction coefficient is supposed to be a constant quantity. A posteriori, this constraint is generally justified by the goodness of the results, but its verification is rarely undertaken. 


\section{Deviations from Bouguer's linear law}

In our observing programme of $\delta$ Sct stars (Poretti and Mantegazza, 1992) we collected a large sample of extinction coefficient behaviours. We used in our analysis the simultaneous least-squares fitting technique proposed by Vaníček (1971) and the power spectra of the magnitude differences between two comparison stars always show an increasing number of peaks at the lowest frequencies: if the peaks are observed at integer values of $c / d$ we ascribe them to meteorological effects. With a large number of measurements, these effects become more evident because in the power spectrum the white noise level decreases and also periodicities (spurious or real) with a very small amplitude can be detected. By analyzing very rich datasets, we noticed that a spurious periodicity can be introduced in the data if, in presence of sky transparency variations, a constant value of $k_{\lambda}^{\prime}$ was used (see below, Fig. 4).

In our observing experience at La Silla we generally found Bouguer's lines with a correlation coefficient very close to 1 , but in September and October 1991 only on 6 nights (out of the 16 with a sufficient time baseline) we observed a constant $k_{B}^{\prime}$ coefficient. Figure 1 shows an example of the behaviour of the instrumental magnitude of HD 225086, a comparison star, in function of $\sec z$ : an interpolation by means of two lines cannot be considered a satisfactory solution because there is no physical reason for an abrupt change in the $k_{B}^{\prime}$ coefficient in correspondance with the meridian crossing. Actually, $k_{B}^{\prime}$ changes continuously during the night.

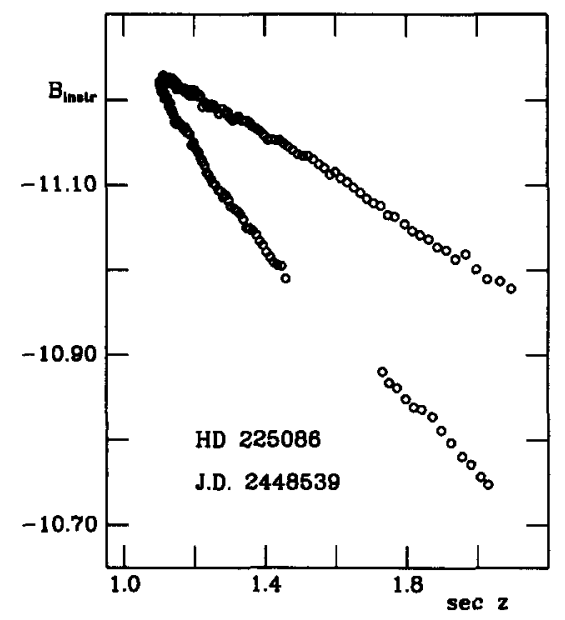

Figure 1. Behaviour of the instrumental magnitude showing the effect of a variable extinction coefficient (ESO measurements).

The non-linear behaviour of the extinction law observed not only at Merate Observatory, but also at such a good photometric site as La Silla gave us the warning 
sign that a method not requiring a constant value of $k_{\lambda}^{\prime}$ should be developed.

\section{Instantaneous determination of the first-order term $k^{\prime}$}

The starting point for the correction of the extinction in presence of a rapid variability of the $k_{\lambda}^{\prime}$ coefficient can be found in the reliability of a photon-counting instrumentation that works in conditions which can be maintained constant over a long time baseline. The constant response to a standard signal (e.g. a constant artificial light source) guarantees that the above conditions are fulfilled.

If the stability is maintained over the whole observing run, the extra-atmosphere instrumental magnitude $m_{o}$ will be a constant quantity. If we can calculate $m_{o}$ for one of the two comparison stars (used as reference star), the extinction coefficient can be easily determined at the time $t$ of the measurement $m(t)$ by means of

$$
k^{\prime}(t)=\frac{m(t)-m_{o}}{\sec z}
$$

The main difficulty in the application of this equation is to have a reliable value for $m_{o}$. A good opportunity is offered by the analysis of the errors resulting from a wrong choice of the value for $m_{0}$. If we can neglect the colour effect, for each measurement of the magnitude difference $\Delta m(t)$ between the two stars we can calculate the extraatmosphere magnitude difference $\Delta m_{o}$ by means of

$$
\Delta m_{o}=\Delta m(t)-\frac{m(t)-m_{o}}{\sec z} \Delta \sec z
$$

where $m(t)$ and $m_{o}$ are the magnitudes of the reference star inside and outside atmosphere. If we are considering an $m_{o}$ value which differs by $\delta$ from the true value $m_{o}^{*}$, we will observe a linear trend when plotting $\Delta m_{o}$ (or the residuals $\Delta m_{o}-\overline{\Delta m_{o}}$ ) against $\Delta \sec z / \sec z$; the angular coefficient yields exactly $\delta$ :

$$
\Delta m_{o}=\Delta m(t)-\frac{m(t)-\left(m_{o}^{*}+\delta\right)}{\sec z} \Delta \sec z=\Delta m(t)-\frac{m(t)-m_{o}^{*}}{\sec z} \Delta \sec z+\delta \frac{\Delta \sec z}{\sec z}
$$

Hence, we can determine the $m_{o}$ value which does not yield any trend in the residuals or, similarly, for the $m_{o}$ value which minimizes the height of the peak at integer values of $\mathrm{c} / \mathrm{d}$ in the power spectrum. Figure 2 shows the satisfactory results obtained by applying this procedure to the 2715 measurements between HD 18768 and HD 19279: for different values of $m_{o}$, the plot of the residuals (left side) displays a progressive change in slope, while the power spectra of the magnitude differences (right side) show the corresponding flattening of the noise at the lowest frequencies. We can conclude that $m_{o}^{*}=-11.75$. It should be noticed that the iterative procedure is not necessary, but we recommend it in order to avoid unreliable determinations. 

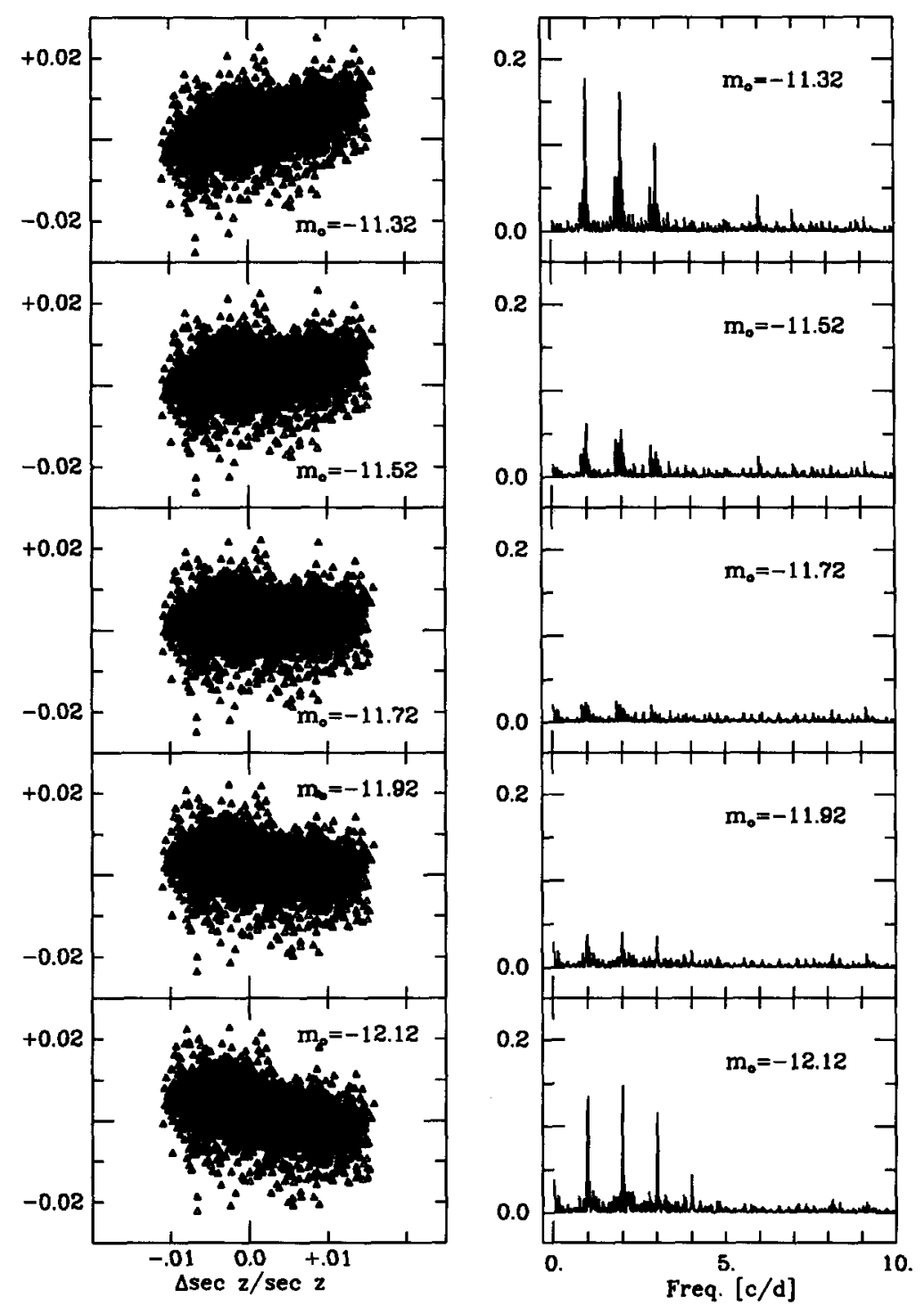

Figure 2. The reduction of the $\Delta V$ measurements between HD 18768 and HD 19278 assuming different values for $m_{o}$. Left panels: the linear trends have an angular coefficient equal to $m_{o}^{*}-m_{o}$. Right panels: the power spectra evidence an increasing number of peaks at the lowest frequencies for increasing values of $\left|m_{o}^{*}-m_{o}\right|$. 
This procedure allows us to reconstruct the behaviour of the $k_{\lambda}^{\prime}$ coefficient. Figure 3 shows the behaviour of the $k_{B}^{\prime}$ and $k_{V}^{\prime}$ coefficients at La Silla: slow, but sometimes large variations are present. $k_{B}^{\prime}$ values are ranging from 0.26 to $0.58 \mathrm{mag} /$ airmass (the maximum amplitude for a night is $0.12 \mathrm{mag} /$ airmass, see J.D. 2448539 and compare the behaviour of the $k_{B}^{\prime}$ coefficient with that of the instrumental magnitude reported in Fig. 1), while $k_{V}^{\prime}$ values are ranging from 0.20 to $0.28 \mathrm{mag} /$ airmass. We notice that these values are slightly higher than the normal ones. Figure 3 also shows that the $k_{\lambda}^{\prime}$ coefficients change slowly, without any well-defined periodicity or behaviour: for this reason any attempt to fit their variability by means of a periodic term cannot solve the problem.

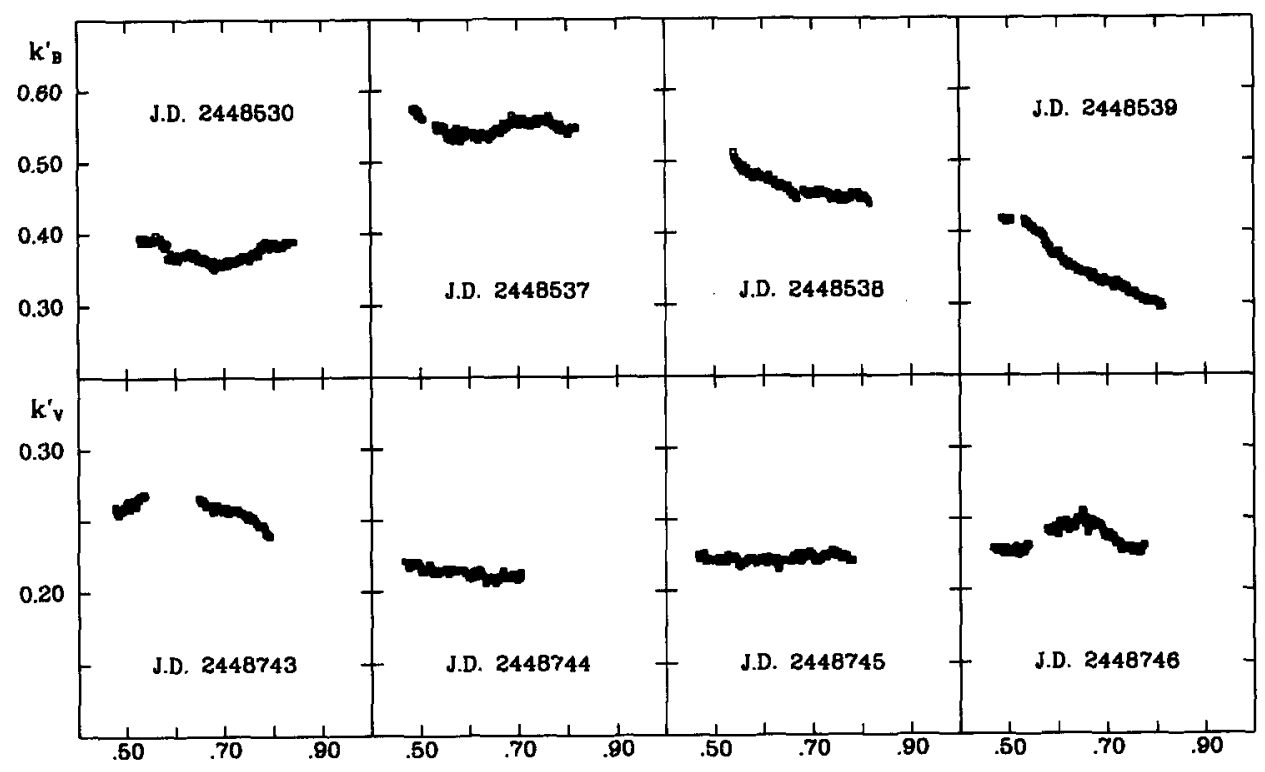

Figure 3. Behaviour of the extinction coefficients $k_{B}^{\prime}$ and $k_{V}^{\prime}$ on some nights at La Silla. It was determined by applying our method to the measurements of HD 225086 ( $B$ light) and HD 105654 ( $V$ light).

\section{Methodological conclusions}

The method described here provides a satisfactory correction of the extinction effect; its application to differential photometry does not require any additional measurement and allows us to clearly recognize spurious periodicities, due to the variability of the $k_{\lambda}^{\prime}$ coefficient during the night. Figure 4 shows the power spectra obtained from the $\Delta V$ measurements between HD 18768 and HD 19279 calculated by assuming 
a constant $k_{V}^{\prime}$ value throughout each night (left panel) and by applying the described procedure (right panel). The height of the spurious peak at $1.001 \mathrm{c} / \mathrm{d}$, well above the noise level in the former case, is strongly reduced in the latter. On the other hand the peak at $14.454 \mathrm{c} / \mathrm{d}$ is present in both spectra and it can be ascribed to the physical variability of HD 19279 .

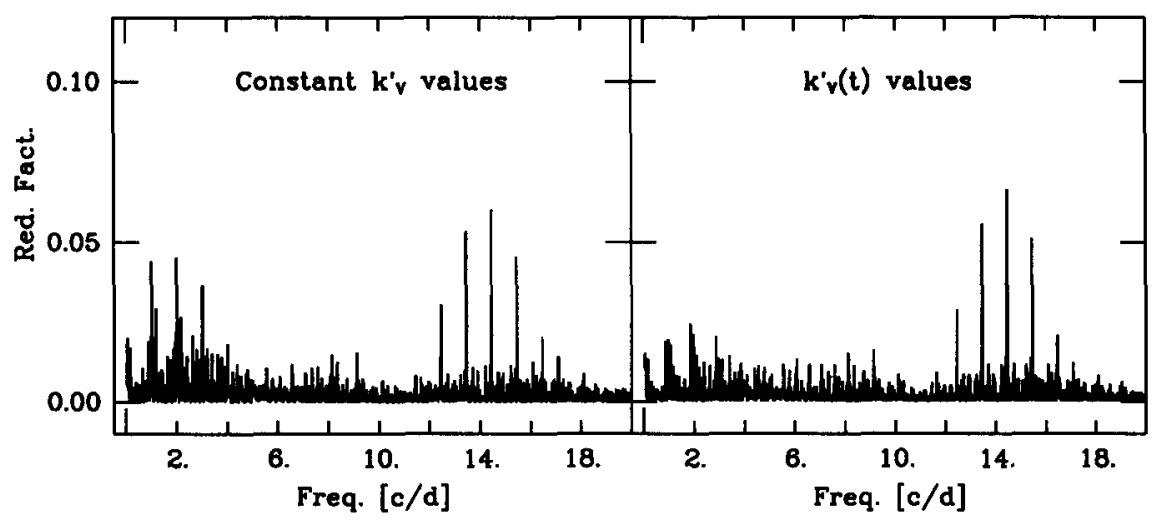

Figure 4. The noise at the lowest frequencies is strongly reduced when using the instan taneous values of $k_{V}$ (right panel) instead of the constant values (left panel).

As regards the colour effect, it is a well known fact that the analysis of the residuals allows us to correct it . It is sufficient to plot the residuals $\Delta m_{o}-\overline{\Delta m_{o}}$ versus $\overline{\sec z}$; if a colour term is present, the angular coefficient of the least-squares line will now give $k^{\prime \prime} \Delta(B-V)$. The two $k_{B}^{\prime \prime}$ values obtained from the ESO measurements are in good agreement with each other: $k_{B}^{\prime \prime}=-0.034 \pm 0.005$ (September and October 1991) and $k_{B}^{\prime \prime}=-0.031 \pm 0.002$ (April 1989).

The extension of the method to standard photometry, where rapid variability of the extinction coefficient can generate erroneous results, can be ensured by the frequent observation of one standard star, which will provide the requested $k_{\lambda}^{\prime}$ behaviour during the night. A particular care should be used when calculating both transformation and extinction coefficients in presence of a variable extinction coefficient since least-squares routines can converge to not-physical values.

The observed evidence of a variable $k_{\lambda}^{\prime}$ coefficient strongly suggests the need of a more rigorous monitoring of the extinction behaviour; volcanic eruptions are the most probable causes for this effect (the effects observed at La Silla are surely related to the eruption of Pinatubo), but the lifetime of dust and aerosol clouds in the Earth's atmosphere does not allow us to consider them as time-limited phenomena.

\section{References:}

Vaníček P., 1971, Astrophys. Space Sci. 12, 10

Poretti E., Mantegazza. L., 1992, The Messenger 68, 33 


\section{Discussion}

R. Shobbrook: How certain are you that your extinction change from east to west is not in fact a drift in gain (due to electronics or telescope orientation)? East to West differences in extinction are not likely at many sites. The absorbing part of the atmosphere in the light path lies only 3 to $5 \mathrm{~km}$ at the most from the observatory so unless the site is on the edge of a major geographical change (e.g. land/water) no effect is likely.

Poretti: My answer to this question is reported in the answer to A. Young's comments.

A. T. Young: In response to Bob Shobbrook's question, the examples shown are very typical for an extinction coefficient that changes linearly with time. A zero-point drift produces a rounded corner, not a sharp angle, in the Bouguer plot. The two slopes, of the rising and setting branches contain aliasing effects due to the correlation of airmass and time, neither slope is a 'real' extinction coefficient.

Poretti: Daily tests should be performed to check instrumental drifts as we did. Moreover, instrumental drifts would be regular. In our case, we observed changing behaviour (and constant ones, too) when observing the same comparison star on different nights; this suggests the atmosphere was responsible for the variability of the instrumental magnitude.

W.Z. Wisniewski: Can the different behaviour of extinction when it is rising and setting be explained by asymmetry between east and west?

Poretti: A continuous drift of the $\mathrm{k}^{\prime}$ coefficient seems to be more plausible than an eastwest dicotomy; see also Bob Shobbrook's comment. Moreover, our method does not show this dicotomy, which, if real, should be observed.

C.L. Sterken: A question to observers at good and less good sites: have you ever experienced any periodicity or quasi-periodicity in extinction coefficients on time scales of about 10 days to a fortnight?

Poretti: Seasonal variability of $\mathrm{k}^{\prime}$ extinction coefficient was recorded in many observatories. We performed frequency analysis of $\mathrm{k}^{\prime}(\mathrm{t})$ values, but we failed to find evidence of well defined periodicities or quasi-periodicities. 